\title{
Updated molecular phylogenetic data for Opisthorchis spp. (Trematoda: Opisthorchioidea) from ducks in Vietnam
}

Thanh Thi Ha Dao ${ }^{1,2,3}$, Thanh Thi Giang Nguyen ${ }^{1,2}$, Sarah Gabriël ${ }^{4}$, Khanh Linh Bui ${ }^{5}$, Pierre Dorny ${ }^{2,3^{*}}$ (D) and Thanh Hoa Le ${ }^{6}$

\begin{abstract}
Background: An opisthorchiid liver fluke was recently reported from ducks (Anas platyrhynchos) in Binh Dinh Province of Central Vietnam, and referred to as "Opisthorchis viverrini-like". This species uses common cyprinoid fishes as second intermediate hosts as does Opisthorchis viverrini, with which it is sympatric in this province. In this study, we refer to the liver fluke from ducks as "Opisthorchis sp. BD2013", and provide new sequence data from the mitochondrial (mt) genome and the nuclear ribosomal transcription unit. A phylogenetic analysis was conducted to clarify the basal taxonomic position of this species from ducks within the genus Opisthorchis (Digenea: Opisthorchiidae).

Methods: Adults and eggs of liver flukes were collected from ducks, metacercariae from fishes (Puntius brevis, Rasbora aurotaenia, Esomus metallicus) and cercariae from snails (Bithynia funiculata) in different localities in Binh Dinh Province. From four developmental life stage samples (adults, eggs, metacercariae and cercariae), the complete cytochrome b (cob), nicotinamide dehydrogenase subunit 1 ( $($ ad 1 ) and cytochrome c oxidase subunit 1 (cox1) genes, and near-complete 18S and partial $28 \mathrm{~S}$ ribosomal DNA (rDNA) sequences were obtained by PCR-coupled sequencing. The alignments of nucleotide sequences of concatenated cob + nad $1+\operatorname{cox} 1$, and of concatenated $185+285$ were separately subjected to phylogenetic analyses. Homologous sequences from other trematode species were included in each alignment.
\end{abstract}

Results: Phylogenetic trees were inferred from concatenated (cob + nad $1+\operatorname{cox} 1)$ nucleotide sequences and combined 18S + 28S nucleotide sequences of five Opisthorchis sp. BD2013 samples and additional reference taxa. Both trees demonstrated the anticipated clustering of taxa within the superfamily Opisthorchioidea, the paraphyly of the genus Opisthorchis and the sister-species relationship of Opisthorchis sp. BD2013 with O. viverrini.

Conclusions: While it is likely that Opisthorchis sp. BD2013 is distinct from O. viverrini, it is clearly a sister taxon of $O$. viverrini within the limited number of Opisthorchis species for which appropriate sequence data are available. The new sequences provided here will assist the diagnosis and the taxonomic clarification of the opisthorchiid species.

Keywords: Mitochondrial gene, Ribosomal transcription unit, Opisthorchis sp. BD2013, Opisthorchiid, 18S rDNA, $28 \mathrm{~S}$ rDNA, Phylogenetic analysis

\footnotetext{
* Correspondence: pdorny@itg.be

${ }^{2}$ Department of Biomedical Sciences, Institute of Tropical Medicine,

Nationalestraat 155, B2000 Antwerp, Belgium

${ }^{3}$ Department of Virology, Parasitology and Immunology, Faculty of Veterinary

Medicine, Ghent University, 133 Salisburylaan, B9820, Merelbeke, Belgium

Full list of author information is available at the end of the article
} 


\section{Background}

The family Opisthorchiidae (Digenea: Opisthorchioidea) consists of 33 genera considered valid including the genera Opisthorchis and Clonorchis, in which O. viverrini, O. felineus and $C$. sinensis are known to infect humans [1]. Humans become infected by eating uncooked cyprinoid fish containing metacercariae. Opisthorchis viverrini has been reported in Central Vietnam, where Binh Dinh and Phu Yen Provinces are highly endemic for human opisthorchiasis [2-4].

In 2013, Dao et al. [5] found adults of an opisthorchiid species in ducks (Anas platyrhynchos) in areas of Binh Dinh Province where there are many human opisthorchiasis cases. This parasite was then given the working name "Opisthorchis viverrini-like", because of its close similarity to $O$. viverrini $[5,6]$. Subsequently, there has been a debate about the identity of this worm. Nawa et al. [7] argued that the duck liver fluke not be O. viverrini, but is most likely O. parageminus that was previously reported from ducks in Vietnam [8-10]. However, Dorny et al. [11] considered that their "Opisthorchis viverrini-like" species exhibited some morphological differences from $O$. parageminus. We now propose to use the working name "Opisthorchis sp. BD2013" instead of the earlier "Opisthorchis viverrini-like".

Molecular phylogenetic/systematic studies are excellent aids for taxonomy [12-15]. Such studies require homologous sequences from as many taxa as possible within the group of interest. In the genus Opisthorchis, a number of genetic markers from complete mitochondrial sequences and the nuclear ribosomal transcription units including, ITS1, ITS2, 18S rDNA and partial 28S rDNA have been generated for O. viverrini, O. felineus and Clonorchis sinensis. These genetic markers have greatly contributed to molecular diagnostic, epidemiological, phylogenetic and evolutionary studies of the species in Opisthorchiidae and trematodes [3, 13, 16-19]. However, Opisthorchis is a very large genus [7], and molecular data are available for only a few species. Moreover, given difficulties with the morphological taxonomy within the genus, it is not always certain that names assigned to samples are accurate. The only molecular data claimed to be from O. parageminus consist of two sequences recently deposited in GenBank (accession numbers KX258656, KX258657) by Nguyen and Nguyen (otherwise unpublished data). Although their worms came from ducks in Vietnam, no information is available on the morphological basis for the identification. Both of these sequences (mitochondrial partial mt cox 1 and nuclear ribosomal ITS2) are very similar to earlier sequences available for Opisthorchis sp. BD2013 published by [5]. Here, we provide additional mitochondrial sequences, i.e. complete cytochrome $b$ $(c o b)$, nicotinamide dehydrogenase subunit 1 (nad1) and cytochrome $c$ oxidase subunit 1 ( $\operatorname{cox} 1)$ genes, and nearcomplete $18 \mathrm{~S}$ rDNA and partial $28 \mathrm{~S}$ rDNA sequences in an effort to better resolve the affinities of Opisthorchis sp. BD2013 within the family Opisthorchiidae and the superfamily Opisthorchioidea.

\section{Methods}

Opisthorchis sp. BD2013 samples collected from the field

Adult specimens and eggs of Opisthorchis sp. BD2013 were collected from naturally infected domestic ducks (Anas platyrhynchos) originating from 4 localities (Phu Cat, Phu My, An Nhon and Tuy Phuoc Districts) in Binh Dinh Province of Central Vietnam [6, 20] (Table 1). Each adult worm, unstained or stained with acetic carmine, was morphologically identified by light microscopy [5]. Up to three adult worms from each locality were individually fixed in $70 \%$ ethanol, and one or two worms from each locality were separately subjected to genomic DNA extraction and molecular analysis.

Fishes (harbouring metacercariae) and snails (shedding cercariae) were collected from My Tho Lake in the lowlands of Binh Dinh Province [20]. Infected fishes were identified as Puntius brevis, Esomus metallicus, Rasbora aurotaenia, and the snail as Bithynia funiculata [20] (Table 1). For molecular analysis, metacercariae and cercariae were individually fixed in RNAlater $^{\mathrm{TM}}$ buffer (Qiagen, Texas, USA) at $4{ }^{\circ} \mathrm{C}$. Individual parasites from each intermediate host and each locality were used for extraction of DNA and molecular study.

Eggs were individually collected from the gallbladder of naturally infected ducks by washing and centrifuging the bile ten times in normal saline $(0.9 \% \mathrm{NaCl})$, then three times in phosphate buffered saline (PBS) before storage at $-20{ }^{\circ} \mathrm{C}$ until use (Table 1 ).

\section{Genomic DNA extraction and primers}

Total genomic DNA was extracted from individual adults, metacercariae, cercariae or pooled eggs (approximately 2000-3000 eggs) using the GeneJET ${ }^{\mathrm{Tm}}$ Genomic DNA Purification Kit (Thermo Fisher Scientific Inc., MA, USA), according to the manufacturer's instructions. A slight modification applied for eggs was to increase the incubation period by 3-4 h after enzymatic lysis. Genomic DNA was eluted in $50 \mu$ of the elution buffer provided in the kit and stored at $-20{ }^{\circ} \mathrm{C}$. The DNA concentration was estimated using a GBC UV/visible 911A spectrophotometer (GBC Scientific Equipment Pty. Ltd., Braeside, Australia) and diluted to a working concentration of $50 \mathrm{ng} / \mu \mathrm{l}$ (about $10 \mathrm{ng} / \mu \mathrm{l}$ for DNA from eggs). From this genomic DNA, 2-3 $\mu$ l was used as template in a PCR of $50 \mu \mathrm{l}$ volume.

Primers used both for amplification and sequencing of the mitochondrial and nuclear ribosomal genes are listed in Table 2. The primer pair OACOBF/OACO1R 
Table 1 List of field samples used in this study, their geographical collection site in Binh Dinh province and their hosts

\begin{tabular}{|c|c|c|c|c|}
\hline Life-cycle stage & Site collected (district) & Host & Scientific name & Sample abbreviation for use in this study \\
\hline Adult worm & Phu Cat & Duck & Anas platyrhynchos & Opisthorchis sp. BD2013-PC6aduBD \\
\hline Adult worm & Phu My & Duck & Anas platyrhynchos & Opisthorchis sp. BD2013-PM10aduBD \\
\hline Adult worm & An Nhon & Duck & Anas platyrhynchos & \\
\hline Adult worm & Tuy Phuoc & Duck & Anas platyrhynchos & \\
\hline Metacercariae & Phu My & Fish & Puntius brevis & Opisthorchis sp. BD2013-PCmetaBD \\
\hline Metacercariae & Phu My & Fish & Rasbora aurotaenia & \\
\hline Metacercariae & Phu My & Fish & Esomus metallicus & \\
\hline Cercariae & Phu My & Snail & Bithynia funiculata & Opisthorchis sp. BD2013-PCcercaBD \\
\hline Eggs & Phu My & Duck & Anas platyrhynchos & Opisthorchis sp. BD2013-PCeggBD \\
\hline
\end{tabular}

amplified approximately $7.8 \mathrm{~kb}$ of mtDNA. Based on the sequence obtained from this amplicon, three primer pairs specific for the individual target proteincoding genes were designed. Primer pairs OACOBF/ OACOBR, OAND1F/OAND1R, OACO1F/OACO1R amplified complete $c o b$, nad 1 and $c o x 1$ genes, respectively. The primer pairs U18SF/U18SR were used for obtaining major fragments of ribosomal $18 \mathrm{~S}$ and U28SF/U28SR for 28S, respectively [12]. Additional internal primers were designed and used as needed (Table 2).

\section{Amplification of mitochondrial and ribosomal genes The $7.8 \mathrm{~kb} \mathrm{mt}$ genomic region}

Long PCR reactions were prepared using $25 \mu \mathrm{l}$ of Fusion High-Fidelity PCR Master Mix $(2 \times)$ (Thermo Fisher Scientific Inc., Waltham, MA, USA) and $2 \mu \mathrm{l}$ of each primer $(10 \mathrm{pmol} / \mu \mathrm{l}), 2 \mu \mathrm{l}$ DNA template of the adult sample $(50 \mathrm{ng} / \mu \mathrm{l}), 2 \mu \mathrm{l}$ DMSO (dimethyl sulfoxide) and $17 \mu \mathrm{l} \mathrm{H}_{2} \mathrm{O}$ up to a final volume of $50 \mu$ l. All PCRs were performed in an MJ PTC-100 thermal cycler with initiation at $98{ }^{\circ} \mathrm{C}$ for $30 \mathrm{~s}$, followed by 35 cycles consisting of denaturation for $10 \mathrm{~s}$ at $98{ }^{\circ} \mathrm{C}$, annealing at $56{ }^{\circ} \mathrm{C}$ for $30 \mathrm{~s}$, extension at $72{ }^{\circ} \mathrm{C}$ for $6 \mathrm{~min}$.

\section{Individual $\mathrm{mt}$ and ribosomal DNA genes}

PCR reactions of $50 \mu \mathrm{l}$ were prepared using $25 \mu \mathrm{l}$ of DreamTaq PCR Master Mix $(2 \times)$ (Thermo Fisher Scientific Inc., Waltham, MA, USA), $2 \mu$ of each primer (10 pmol/ $\mu \mathrm{l}), 2 \mu \mathrm{l}$ DNA template $(50 \mathrm{ng} / \mu \mathrm{l}$ for adults; $50 \mathrm{ng} / \mu \mathrm{l}$ for metacercariae; $10-20 \mathrm{ng} / \mu \mathrm{l}$ for cercariae and eggs), $2 \mu \mathrm{l}$ DMSO (dimethyl sulfoxide) and $17 \mu \mathrm{l}$ $\mathrm{H}_{2} \mathrm{O}$. All PCRs were performed in an MJ PTC-100 thermal cycler with initiation at $94{ }^{\circ} \mathrm{C}$ for $5 \mathrm{~min}$, followed by 35 cycles consisting of denaturation for $30 \mathrm{~s}$ at $94{ }^{\circ} \mathrm{C}$, annealing at $56{ }^{\circ} \mathrm{C}$ for $30 \mathrm{~s}$, extension at $72{ }^{\circ} \mathrm{C}$ for $3 \mathrm{~min}$.

Table 2 Primers for amplification and sequencing of the mitochondrial protein-coding and nuclear ribosomal genes used in this study

\begin{tabular}{|c|c|c|c|c|c|}
\hline Primer name & Sequence $\left(5^{\prime}-3^{\prime}\right)$ & Target gene & Amplicon by PCR & Length of sequence (bp) & Reference \\
\hline OACOBF & AGCCGGAGAGTCATTGTGTG & $c o b$ & $1.4 \mathrm{~kb}$ & 1110 & This study \\
\hline OACOBR & TGAATCCCACAACCGCGTTA & & & & \\
\hline $\mathrm{OACOBR}^{\mathrm{a}}$ & TACGTTGAAGGACGGGTTGG & & & & \\
\hline OAND1F & CGTGTGGTGGGGCAAGATAG & nad 1 & $1.2 \mathrm{~kb}$ & 903 & This study \\
\hline OAND1R & CCACACAGCCTTCTCAAGGT & & & & \\
\hline OACO1F & GAGGGTTACGTGGGTTGGAG & $\operatorname{cox} 1$ & $1.8 \mathrm{~kb}$ & 1551 & This study \\
\hline OACO1R & CAACCCTACTAAGCACCACAGC & & & & \\
\hline $\mathrm{OACO}_{\mathrm{R}} 2^{\mathrm{a}}$ & GGATCCCAAAAACGCTCACG & & & & \\
\hline U18SF & GCGAATGGCTCATTAAATCAGC & $18 \mathrm{~S}$ & $1.8 \mathrm{~kb}$ & $\sim 1790$ & [12] \\
\hline U18SR & GGAACCAATCCGAGGACCTTGC & & & & \\
\hline $\mathrm{NS} 2 \mathrm{~F}^{\mathrm{a}}$ & GCAAGTCTGGTGCCAGCAGCC & & & & \\
\hline U28SF & CTAACAAGGATTCCCTTAGTAAC & 285 & $1.3 \mathrm{~kb}$ & $\sim 1100$ & [12] \\
\hline U28SR & GTCTTTCGCCCCTATACTCAC & & & & \\
\hline
\end{tabular}




\section{Sequencing and sequence analyses}

PCR products were obtained from at least two individual samples for each template (i.e. adults, metacercariae, cercariae and eggs) originating from different geographical localities. The PCR products $(10 \mu \mathrm{l}$ of each) were examined on a $1 \%$ agarose gel, stained with ethidium bromide, and visualized under UV light (Wealtec, Meadowvale Way Sparks, USA).

All the purified or gel-extracted amplicons were subjected to direct sequencing by automated sequencers using amplifying/flanking and internal primers (Table 2) by primer-walking in both directions (Macrogen Inc., Seoul, South Korea). Sequences (two from each sample) were aligned to obtain the final sequence for characterization. All sequences of Opisthorchis sp. BD2013 were identical, regardless of the life-cycle stage or locality.

The concatenated nucleotide and amino acid sequences of three protein-coding genes, i.e., $c o b+n a d 1+\operatorname{cox} 1$, were used to infer the pairwise genetic distances between 10 opisthorchiids (Table 3). These isolates included Opisthorchis sp. BD2013 and the reference sequences from Laos (JF739555), Vietnam (MF287777-MF287779) and Thailand (MF287780-MF287782). The genetic distances were inferred by pairwise analysis using the MEGA6.0 software, and the number of base substitutions per site was calculated by the most simplified method (uncorrected p-distance) [21].

\section{Phylogenetic analysis \\ Preparation of DNA sequences}

Phylogenetic analysis using three mitochondrial proteincoding (cob, nad1, cox1) and two nuclear ribosomal (18S and $28 \mathrm{~S} \mathrm{rDNA}$ ) genes was conducted to examine the taxonomic placement of Opisthorchis sp. BD2013 from ducks within the superfamily Opisthorchioidea. Sequences of trematode species/isolates of the Opisthorchiidae, Heterophyidae, Fasciolidae and Schistosomatidae (as the outgroup) were used. Summary data of species/ isolates, mainly from the available complete mitochondrial genomes are presented in Table 3. Accession numbers for the target and reference $18 \mathrm{~S}$ and $28 \mathrm{~S}$ rDNA sequences are listed in Table 4. For Opisthorchis sp. BD2013, we decided to use only two sequences of adults, and one each from metacercariae, cercariae and eggs for phylogenetic analyses.

Concatenated nucleotide sequences of $\mathrm{mt}$ proteincoding genes (cob, nad1, cox 1$)$ from adults, metacercariae, cercariae, and eggs of Opisthorchis sp. BD2013, and from additional taxa (available in GenBank; see Table 3) were imported into GENEDOC 2.7 (available at http://iubio.bio.indiana.edu/soft/molbio/ibmpc/genedoc-readme.html) and aligned for phylogenetic analysis. Additionally, the sequences of opisthorchiids were translated (using the echinoderm/flatworm mitochondrial genetic code: translation Table 9 in GenBank), and the deduced amino acid sequences were aligned for pairwise genetic distance analysis.

DNA sequences of $18 \mathrm{~S}$ rRNA and $28 \mathrm{~S}$ rRNA genes (listed in Table 4) were aligned separately using GENEDOC 2.7. The sequences were trimmed at both ends to the shortest length of the representative sequences. For $18 \mathrm{~S}$ rDNA, in this study, the final alignment was 2005 nucleotides (nt) long of which 87 nt positions were trimmed at $5^{\prime}$ end and 114 nt at $3^{\prime}$ end, leaving 1804 characters for analyses. For $28 \mathrm{~S}$ rDNA, the final alignment was1449 nt long of which 122 nt positions were trimmed at $5^{\prime}$ end and 123 nt at $3^{\prime}$ end, leaving 1202 characters for analyses. The two sequences were then concatenated as indicated in Table 4, preferably from the same strains/isolates. The concatenated $18 \mathrm{~S}+28 \mathrm{~S}$ rDNA sequences representing species/isolates were imported into GENEDOC 2.7 and phylogenetic analysis and tree construction were done by MEGA6.0 [21].

\section{Phylogenetic reconstruction}

The alignments of the concatenated nucleotide ( $c o b$, cox 1 , nad 1 ) and $18 \mathrm{~S}+28 \mathrm{~S}$ sequences, respectively, were trimmed to the length of the shortest sequence and imported into the MEGA 6.06 software [21]. Maximum likelihood (ML) analyses were performed in each case. For DNA sequences, we used the general time-reversible model of evolution with gamma distributed rate heterogeneity and a proportion of invariant sites (GTR $+\Gamma+$ I). This model was given the best Bayesian information criterion score by MEGA. For amino acid sequences, the Jones-Taylor-Thornton (JTT) model with uniform rates and Nearest-Neighbor-Interchange (NNI) method was used. The confidence in each node was assessed using 1000 bootstrap resamplings [21].

\section{Results \\ Mitochondrial cob, nad1, cox 1 and genetic distances among opisthorchiid species/sequences}

For Opisthorchis sp. BD2013, lengths of the complete cob, nad 1 and cox 1 genes were 1110, 903 and $1551 \mathrm{nu}-$ cleotides, respectively. Among opisthorchiid species, $c o b$ genes ranged in length from 1110 to $1116 \mathrm{nt}$, and cox 1 genes were 1551 to $1563 \mathrm{nt}$ in length. The primer pairs U18SF/U18SR were used for obtaining major fragments of ribosomal $18 \mathrm{~S}$ and U28SF/U28SR for $28 \mathrm{~S}$ rDNA.

Nucleotide and amino acid pairwise comparisons of the concatenated $\mathrm{mt}$ genes among ten opisthorchiid isolates/species are presented in Tables 5 and 6 . The concatenated $c o b+n a d 1+c o x 1$ nucleotide sequences of Opisthorchis sp. BD2013 differed at 14.4-14.5\% of nucleotide sites and $10.3-10.6 \%$ of amino acid positions from the reference sequences of $O$. viverrini (Vietnam, 
Table 3 Summary data for complete mitochondrial genomes of species providing cytochrome $b$ (cob), nicotinamide dehydrogenase subunit 1 (nad1) and cytochrome coxidase subunit 1 (cox1) used in the phylogenetic analysis including Opisthorchis sp. BD2013 in ducks in Vietnam

\begin{tabular}{|c|c|c|c|c|}
\hline Family/Species & Isolates/Strains & Country & GenBank ID & Reference \\
\hline \multicolumn{5}{|l|}{ Opisthorchiidae } \\
\hline Opisthorchis sp. BD2013 & PC6aduBD & Vietnam & MF287762-MF287764 & This study \\
\hline Opisthorchis sp. BD2013 & PM10aduBD & $V_{i e t n a m}^{b}$ & MF287765-MF287767 & This study \\
\hline Opisthorchis sp. BD2013 & PCmetaBD & Vietnam & MF287768-MF287770 & This study \\
\hline Opisthorchis sp. BD2013 & PCcercaBD & Vietnam & MF287771-MF287773 & This study \\
\hline Opisthorchis sp. BD2013 & PCeggBD & Vietnam & MF287774-MF287776 & This study \\
\hline \multirow[t]{3}{*}{ Opisthorchis viverrini } & na & Laos $^{b}$ & JF739555 & [19] \\
\hline & Binh Dinh 1 & $V_{i e t n a m}{ }^{b}$ & MF287777-MF287779 & This study \\
\hline & Khon Kaen & Thailand $^{\mathrm{b}}$ & MF287780-MF287782 & This study \\
\hline Opisthorchis felineus & Ust-Tula (Novosibirsk) & Russiab $^{\mathrm{b}}$ & EU921260 & {$[16]$} \\
\hline \multirow[t]{4}{*}{ Clonorchis sinensis } & Nam Dinh & Vietnam $^{c}$ & MF287783-MF287785 & This study \\
\hline & Guangdong & China $^{\mathrm{b}}$ & JF729303 & [19] \\
\hline & na & South Koreab & JF729304 & [19] \\
\hline & Amur - Khabarovsk & Russiab $^{\mathrm{b}}$ & FJ381664 & {$[16]$} \\
\hline Metorchis orientalis & Heilongjiang & China $^{b}$ & KT239342 & {$[22]$} \\
\hline \multicolumn{5}{|l|}{ Heterophyidae } \\
\hline \multirow[t]{2}{*}{ Haplorchis taichui } & na & Laos & KF214770 & {$[24]$} \\
\hline & Quang Tri 3 & Vietnam & MF287786-MF287788 & This study \\
\hline Metagonimus yokogawai & na & South Korea & KC330755 & \\
\hline \multicolumn{5}{|l|}{ Fasciolidae } \\
\hline Fasciola hepatica & Geelong & Australia & AF216697 & {$[25]$} \\
\hline \multirow[t]{2}{*}{ Fasciola gigantica } & Guangxi & China & KF543342 & {$[26]$} \\
\hline & Thua Thien-Hue & Vietnam & MF287789-MF287791 & This study \\
\hline Fasciola sp. (intermediate form) & GHL-Heilongjiang & China & KF543343 & {$[26]$} \\
\hline \multirow[t]{2}{*}{ Fasciolopsis buski } & Jiangxi & China & KX169163 & {$[27]$} \\
\hline & Ha Tay & Vietnam & MF287792-MF287794 & This study \\
\hline Fascioloides magna & Kokořínsko & Czech Republic & KU060148 & {$[28]$} \\
\hline \multicolumn{5}{|l|}{ Schistosomatidae } \\
\hline Schistosoma haematobium ${ }^{a}$ & N10 Village & Mali & DQ157222 & {$[29]$} \\
\hline
\end{tabular}

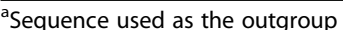

bequences of the opisthorchiids used for pairwise genetic distance calculation (Tables 5 and 6)

Thailand and Laos isolates) [19]; $17.9-18.2 \%$ for nucleotides and $13.3-13.7 \%$ for amino acids from C. sinensis (Russia, China, South Korea and Vietnam isolates); $18.1 \%$ (nucleotides) and $13.7 \%$ (amino acids) from $O$. felineus (a Russian isolate) [16] and 15.4\% (nucleotides) and $11.6 \%$ (amino acids) from Metorchis orientalis (China isolate) [23].

Within each opisthorchiid taxon, pairwise genetic distances were small, only $0.4-0.7 \%$ for nucleotides and $0.5-0.6 \%$ for amino acids within O. viverrini; $0.3-0.6 \%$ (nucleotides) and $0.2-0.8 \%$ (amino acids) within $C$. sinensis. Opisthorchis sp. BD2013 in ducks differs from O. viverrini by more than $10 \%$, a figure comparable to those separating species within the genus Opisthorchis and the family Opisthorchiidae (Tables 5 and 6).

\section{Phylogenetic analysis}

Phylogenetic reconstruction based on the complete cob + nad $1+$ cox 1 amino acid sequences

A phylogenetic tree was constructed from 25 nucleotide sequences inferred from complete $c o b+n a d 1+\operatorname{cox} 1$ of 13 trematode species belonging to 4 families with 
Table 4 Accession numbers of the reference 185 and $28 \mathrm{~S}$ rDNA sequences and their species information used for phylogenetic analysis with those derived from Opisthorchis sp. BD2013 in ducks in the present study

\begin{tabular}{|c|c|c|c|c|}
\hline Family/Species & $18 \mathrm{~S}$ rDNA GenBank ID (isolate) ${ }^{b}$ & $28 \mathrm{~S}$ rDNA GenBank ID (isolate) ${ }^{\mathrm{b}}$ & Origin of sequences & Reference \\
\hline \multicolumn{5}{|l|}{ Opisthorchiidae } \\
\hline \multirow[t]{5}{*}{ Opisthorchis sp. } & MF077358 (PC6aduBD) & MF110001 (PC6aduBD) & Vietnam & This study \\
\hline & MF077359 (PCcercaBD) & MF110002 (PCcercaBD) & Vietnam & This study \\
\hline & MF077360 (PCeggBD) & MF110003 (PCeggBD) & Vietnam & This study \\
\hline & MF077361 (PCmetaBD) & MF110004 (PCmetaBD) & Vietnam & This study \\
\hline & MF077362 (PM10aduBD) & MF110005 (PM10aduBD) & Vietnam & This study \\
\hline \multirow[t]{4}{*}{ Opisthorchis viverrini } & HM004211 (SK) & HM004188 (SK); & Thailand & {$[30]$} \\
\hline & JF823987 (THASK) & JF823990 (THASK) & Thailand & {$[17]$} \\
\hline & MF077364 (PY2) & MF099792 (PY2) & Vietnam & GenBank \\
\hline & MF077363 (BD1) & KY369165 (BD1) & Vietnam & GenBank \\
\hline Opisthorchis felineus & MF077357 (Ust-Tula) & MF099790 (Ust-Tula) & Russia & GenBank \\
\hline \multirow[t]{3}{*}{ Clonorchis sinensis } & JF823988 (VNM) & JF823989 (VNM) & Vietnam & {$[30]$} \\
\hline & $J F 314770$ (GD) & JF823989 (VNM) & China; Vietnam & GenBank; [30] \\
\hline & MF077353 (NH) & MF099784 (NH) & Vietnam & GenBank \\
\hline \multicolumn{5}{|l|}{ Heterophyidae } \\
\hline \multirow[t]{2}{*}{ Haplorchis pumilio } & HM004194 (HpNP1) & HM004186 (HpNP1) & Thailand & [18] \\
\hline & KX815125 (HPU8) & KX815125 (HPU8) & Vietnam & [12] \\
\hline \multirow[t]{2}{*}{ Haplorchis taichui } & KX815126 (QT3) & KX815126 (QT3) & Vietnam & {$[12]$} \\
\hline & HM004201 (NA3) & HM004187 (NA3) & Thailand & {$[30]$} \\
\hline \multirow[t]{2}{*}{ Haplorchis yokogawai } & HM004207 (CP1) & HM004178 (CP1) & Thailand & [18] \\
\hline & HM004208 (CP2) & KY369160 (An394) & Thailand; Vietnam & {$[12,18]$} \\
\hline \multirow[t]{2}{*}{ Procerovum varium } & HM004199 (PvNP1) & HM004182 (PvNP1) & Thailand & {$[30]$} \\
\hline & MF077365 (HspND) & KY369161 (HspND) & Vietnam & GenBank; [12] \\
\hline \multirow[t]{2}{*}{ Stellantchasmus falcatus } & HM004202 (VN1) & HM004174 (VN1) & Vietnam & [17] \\
\hline & MF077366 (QN2) & KY369164 (QN2) & Vietnam & [12] \\
\hline Metagonimus takahashii & HQ832629 (Mt3) & HQ832638 (Mt3) & Japan & {$[31]$} \\
\hline Metagonimus yokogawai & HQ832630 (My1) & HQ832639 (My1) & Japan & {$[31]$} \\
\hline Metagonimus miyatai & HQ832626 (Mm3) & HQ832635 (Mm3) & Japan & [31] \\
\hline \multicolumn{5}{|l|}{ Fasciolidae } \\
\hline Fasciolopsis buski & AY311386 (Vinh) & EU025870 (NA) & Vietnam & {$[32]$} \\
\hline Fasciola gigantica & MF077354 (NB) & MF099787 (NB) & Vietnam & GenBank \\
\hline Fasciola hepatica & MF077355 (Geelong) & MF099788 (Geelong) & Australia & GenBank \\
\hline Fascioloides magna & EF051080 & EU025872 & United States & GenBank; [33] \\
\hline \multicolumn{5}{|l|}{ Schistosomatidae } \\
\hline Schistosoma haematobium $^{a}$ & Z11976 & AY157263 & Mali & {$[34,35]$} \\
\hline
\end{tabular}

${ }^{a}$ Sequence used as the outgroup

${ }^{\mathrm{b}}$ Abbreviations for isolates are given in parentheses

Schistosoma haematobium of the Schistosomatidae as the outgroup (Table 3, Fig. 1). The superfamily Opisthorchioidea in this study comprises the Heterophyidae and Opisthorchiidae (no appropriate sequences from the third family, Cryptogonimidae, were available), with the strong nodal support of $99 \%$, clearly separate from the family Fasciolidae. The Opisthorchis sp. BD2013 clade was placed as a sister of $O$. viverrini from 
Table 5 Pairwise genetic distances (\%) between Opisthorchis sp. BD2013 sample from ducks in Vietnam and the sequences for 0. viverrini, Clonorchis sinensis, O. felineus and Metorchis orientalis of the concatenated mitochondrial genes cob, nad 1 and cox 1

\begin{tabular}{|c|c|c|c|c|c|c|c|c|c|c|c|c|}
\hline & Species & GenBank ID & 1 & 2 & 3 & 4 & 5 & 6 & 7 & 8 & 9 & 10 \\
\hline 1 & Opisthorchis sp. BD2013 (PM10aduBD/Nietnam) & MF287767 & - & & & & & & & & & \\
\hline 2 & O. viverrini (Binh Dinh 1/ Vietnam) & MF287779 & 14.4 & - & & & & & & & & \\
\hline 3 & O. viverrini (Khon Kaen/ Thailand) & MF287782 & 14.5 & 0.4 & - & & & & & & & \\
\hline 4 & O. viverrini (Laos) & $J F 739555$ & 14.4 & 0.5 & 0.7 & - & & & & & & \\
\hline 5 & C. sinensis (Amur-Khabarovsk/Russia) & FJ381664 & 17.9 & 18.1 & 18.1 & 17.9 & - & & & & & \\
\hline 6 & C. sinensis (Guangdong/ China) & JF729303 & 18.0 & 18.1 & 18.1 & 17.9 & 0.4 & - & & & & \\
\hline 7 & C. sinensis (South Korea) & JF729304 & 18.2 & 18.2 & 18.3 & 18.0 & 0.5 & 0.3 & - & & & \\
\hline 8 & C. sinensis (Nam Dinh/Vietnam) & MF287784 & 18.0 & 18.1 & 18.2 & 18.0 & 0.5 & 0.5 & 0.6 & - & & \\
\hline 9 & O. felineus (Ust-Tula/ Russia) & EU921260 & 18.1 & 18.8 & 18.9 & 18.7 & 15.4 & 15.6 & 15.8 & 15.5 & - & \\
\hline 10 & Metorchis orientalis (Heilongjiang/China) & KT239342 & 15.5 & 13.7 & 13.7 & 13.5 & 17.0 & 17.2 & 17.2 & 17.0 & 16.8 & - \\
\hline
\end{tabular}

Thailand, Vietnam and Laos. The genus Opisthorchis appeared as paraphyletic with respect to C. sinensis, O. felineus and M. orientalis (Fig. 1).

\section{Phylogenetic reconstruction based on partial $185+285$ sequences}

Five concatenated $18 \mathrm{~S}+28 \mathrm{~S}$ sequences of Vietnamese Opisthorchis sp. BD2013 (from eggs, cercariae, metacercariae and adults) were aligned with 26 available sequences representing 17 trematode species of the Opisthorchiidae, Heterophyidae, Fasciolidae and Schistosomatidae (outgroup) (Table 4). The nuclear ribosomal dataset from the Opisthorchioidea included available sequences of the $18 \mathrm{~S}+28 \mathrm{~S}$ of 12 taxa only from the Opisthorchiidae and Heterophyidae (data from the Cryptogonimidae were not available). The combined length of alignment in use was between 2940 and 2960 nt. The inferred phylogenetic tree (Fig. 2) again placed Opisthorchis sp. BD2013 in a sister position with $O$. viverrini from Thailand and Vietnam. Again, the genus Opisthorchis appeared as paraphyletic. Monophyly of the superfamily Opisthorchioidea was strongly supported (Fig. 2).

\section{Discussion}

In this study, we used two concatenated datasets to infer the molecular phylogenetic position of Opisthorchis sp. BD2013 (formerly named "Opisthorchis viverrini-like" or as $O$. parageminus by several authors). We did not have samples of O. lobatus [17] and the so-called O. parageminus $[8,9]$ for analysis in the present study, therefore, we were not able to establish the relationship between Opisthorchis sp. BD2013 and these species.

The genus Opisthorchis is very large [7], but relevant sequence data are limited to only a few species. It was necessary to determine whether Opisthorchis sp. BD2013 from ducks is distinct from $O$. viverrini, a zoonotic liver fluke known to infect and to cause cholangiocarcinoma in humans [23]. The data presented in this study strongly imply that the two are distinct species. The sister-species relationship demonstrated between Opisthorchis sp. BD2013, and O. viverrini might simply be because $O$. felineus is the only other member of the genus for which data are available. Opisthorchis felineus renders Opisthorchis paraphyletic in our trees, indicating that much systematic work remains to be done in the

Table 6 Pairwise genetic distances (\%) between Opisthorchis sp. BD2013 sample from ducks in Vietnam and O. viverrini, Clonorchis sinensis, $O$. felineus and Metorchis orientalis of the concatenated mitochondrial amino acid sequence of cob, nad 1 and cox 1

\begin{tabular}{|c|c|c|c|c|c|c|c|c|c|c|c|c|}
\hline & Nucleotide sequences & Accession No. & 1 & 2 & 3 & 4 & 5 & 6 & 7 & 8 & 9 & 10 \\
\hline 1 & Opisthorchis sp. BD2013 (PM10aduBD/Vietnam) & MF287767 & - & & & & & & & & & \\
\hline 2 & O. viverrini (Binh Dinh 1/ Vietnam) & MF287779 & 10.6 & - & & & & & & & & \\
\hline 3 & O. viverrini (Khon Kaen/ Thailand) & MF287782 & 10.6 & 0.5 & - & & & & & & & \\
\hline 4 & O. viverrini (Laos) & $J F 739555$ & 10.3 & 0.6 & 0.6 & - & & & & & & \\
\hline 5 & Clonorchis sinensis (Amur-Khabarovsk/Russia) & FJ381664 & 13.3 & 12.4 & 12.4 & 12.4 & - & & & & & \\
\hline 6 & C. sinensis (Guangdong/ China) & JF729303 & 13.5 & 12.8 & 12.8 & 12.8 & 0.3 & - & & & & \\
\hline 7 & C. sinensis (South Korea) & JF729304 & 13.7 & 12.7 & 12.7 & 12.7 & 0.3 & 0.2 & - & & & \\
\hline 8 & C. sinensis (Nam Dinh/ Vietnam) & MF287784 & 13.6 & 12.6 & 12.6 & 12.6 & 0.4 & 0.8 & 0.8 & - & & \\
\hline 9 & O. felineus (Ust-Tula/ Russia) & EU921260 & 13.7 & 13.8 & 13.9 & 13.9 & 9.3 & 9.7 & 9.7 & 9.5 & - & \\
\hline 10 & Metorchis orientalis (Heilongjiang/China) & KT239342 & 11.6 & 8.8 & 8.8 & 8.7 & 9.8 & 10.2 & 10.2 & 10.1 & 11.0 & - \\
\hline
\end{tabular}




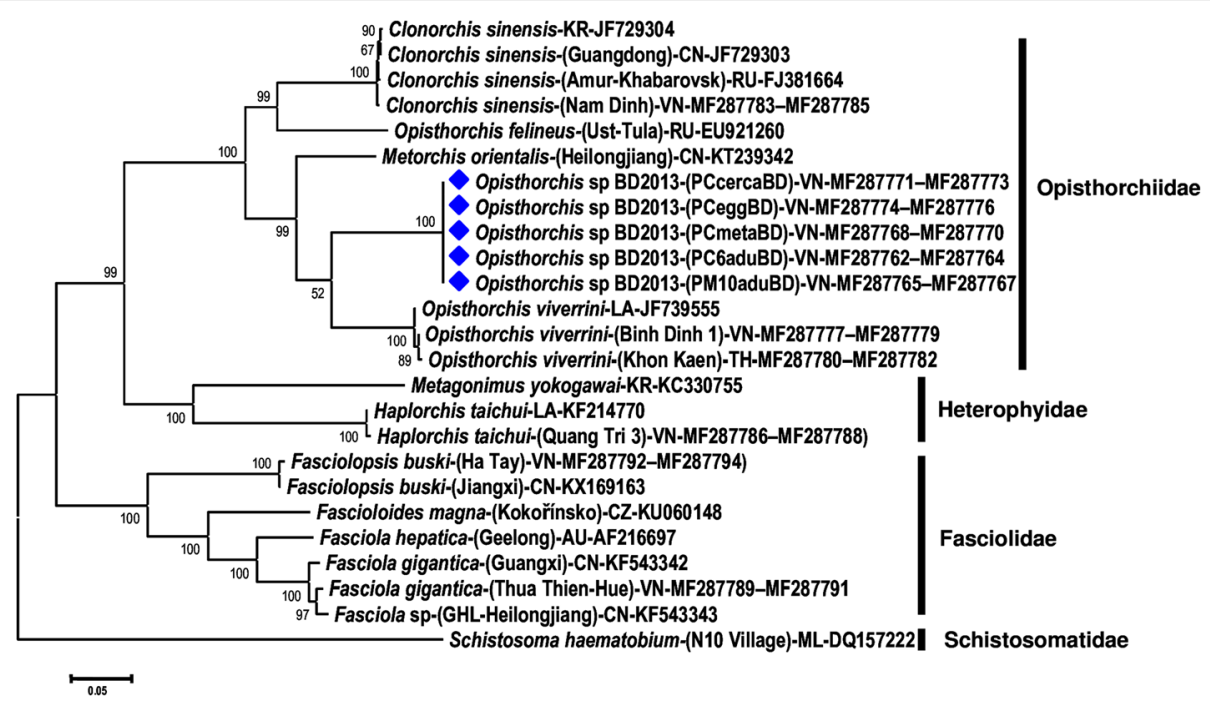

Fig. 1 Phylogenetic tree for Opisthorchis sp. BD2013 (indicated by diamond symbol) and other opisthorchiids and representative trematodes from 4 families, the Opisthorchiidae, Heterophyidae, Fasciolidae and Schistosomatidae (the latter used as an outgroup), based on concatenated nucleotide sequences of complete cytochrome b (cob), nicotinamide dehydrogenase subunit 1 (nad1) and cytochrome $c$ oxidase subunit 1 (cox1) genes. Phylogenetic reconstruction was performed using maximum likelihood analysis with the general time-reversible model with a gamma distributed rate heterogeneity and a proportion of invariant sites $(G T R+\Gamma+I)$ in the MEGA6.06 software package. Support for each node was evaluated using 1000 bootstrap resamplings [21]. The scale-bar indicates the number of substitutions per site. Accession numbers (where available) are given at the end of each sequence name. Isolates/geographical localities are given in parentheses (if available). Country abbreviation codes (2-letter) given prior to the accession numbers: AU, Australia; CN, China; CZ, Czech Republic; KR, Korea; LA, Lao PDR; RU, Russia; TH, Thailand; VN, Vietnam

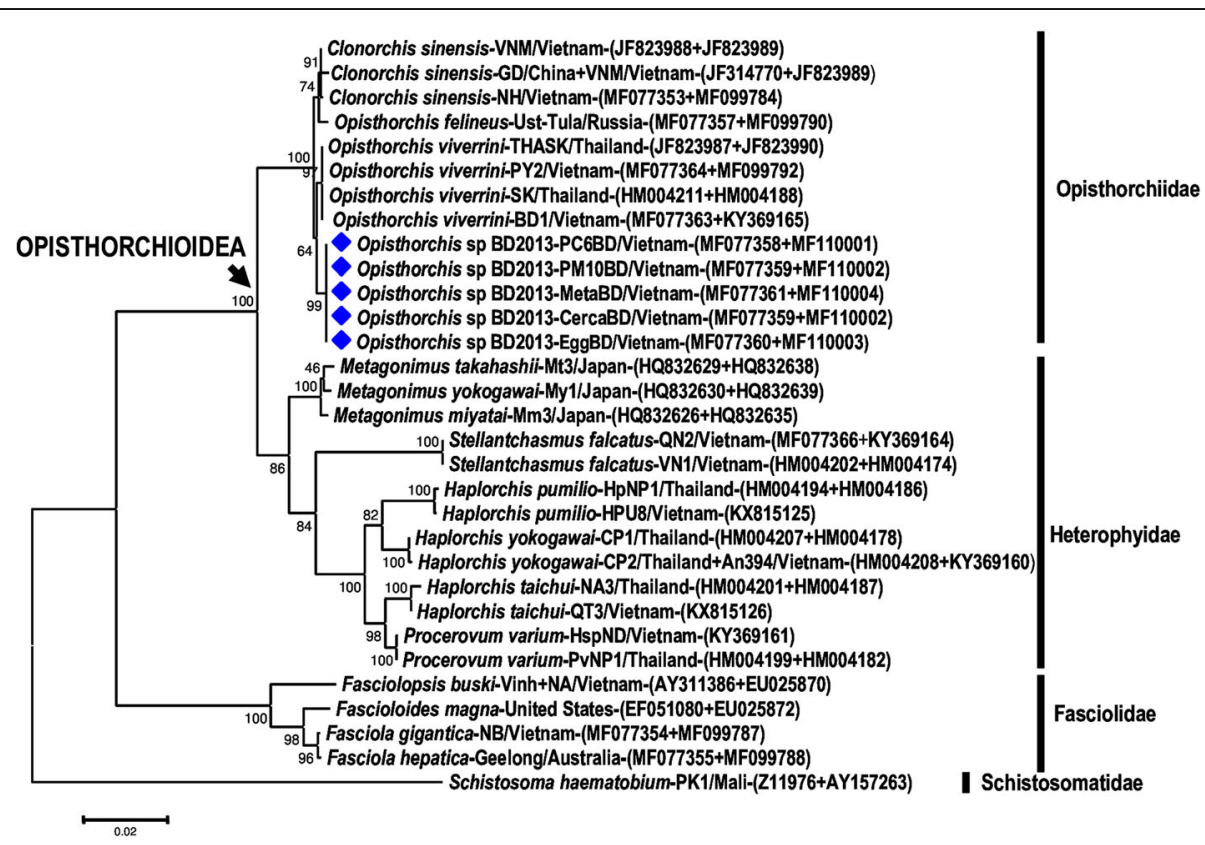

Fig. 2 Phylogenetic tree for Opisthorchis sp. BD2013 (indicated by diamond symbol) and other opisthorchiids and representative trematodes from 4 families, the Opisthorchiidae, Heterophyidae, Fasciolidae and Schistosomatidae (the latter used as the outgroup), based on combined nucleotide sequences of the nuclear small ribosomal subunit (18S rDNA) and large ribosomal subunit (28S rDNA). Phylogenetic reconstruction was performed using maximum likelihood analysis with the general time-reversible model and a gamma distributed rate heterogeneity and proportion of invariant sites (GTR + $\Gamma+\mathrm{I})$ in the MEGA6.06 software package. Support for each node was evaluated using 1000 bootstrap resamplings [21]. The node for the superfamily (infraorder) Opisthorchioidea is indicated by an arrow. The scale-bar indicates the number of substitutions per site. Accession numbers are given at the end of each sequence name. Isolates or geographical localities and country isolated are given in the between (if available) 
Opisthorchiidae. A further unresolved question is the relationship between Opisthorchis sp. BD2013 and O. parageminus. Both were found in ducks in Vietnam, but some morphological differences seem to exist [11]. At this stage, we prefer to leave the question open, pending future morphological and molecular work.

Our previous phylogenetic analysis using short sequences of ITS2 and cox1 revealed close affinities between O. viverrini, O. lobatus and Opisthorchis sp. BD2013 [5]. In the current study, we are unable to resolve the status of O. lobatus compared to Opisthorchis sp. BD2013 and other opisthorchiids.

\section{Conclusions}

Based on mitochondrial $c o b+n a d 1+\operatorname{cox} 1$ and ribosomal $18 \mathrm{~S}+28 \mathrm{~S}$ rRNA sequence analyses, Opisthorchis sp. BD2013 was distinct from O. viverrini, although the two species are closely related. The genus Opisthorchis itself appears as paraphyletic. Data from additional Opisthorchis species are vital to create a phylogeny with higher resolution within Opisthorchis and the Opisthorchiidae.

\section{Abbreviations}

cob: cytochrome b; cox1: cytochrome c oxidase subunit 1; MEGA: Molecular Evolutionary Genetics Analysis; ML: maximum likelihood; mt: mitochondrial; nad1: nicotinamide dehydrogenase subunit 1; rTU: ribosomal transcription unit

\section{Acknowledgments}

We express our thanks to colleagues and technicians at the Binh Dinh Provincial station of veterinary services for providing and processing samples and Ms TK Nguyen and Dr. HTT Doan of the Institute of Biotechnology, Hanoi, Vietnam for contributing to our laboratory work. We would like to express our gratitude to Professor David Blair, College of Science and Engineering, James Cook University, Townsville, Australia for revision and invaluable comments on this paper.

\section{Funding}

This work was funded by the Directorate General for Development Cooperation (DGD) Belgium, through the individual PhD Programme (THT Dao) of the Institute of Tropical Medicine Belgium under the DGD-ITM Framework agreements 3 and 4

\section{Availability of data and materials}

The data sets supporting the alignment and phylogenetic analysis are included in the article. Nucleotide sequences obtained in the present study have been deposited into the GenBank database with the following accession numbers: MF077358-MF077362 (18S rDNA; Opisthorchis sp. BD2013); MF110001-MF110005 (28S rDNA; Opisthorchis sp. BD2013); MF287762-MF287776 (cob, nad1, cox1; Opisthorchis sp. BD2013).

\section{Authors' contributions}

THTD, PD and THL conceived the study, analyses of final data and wrote the manuscript. TGTN, KLB and SG conducted field collections, laboratory, and preliminary sequence analyses. All authors read and approved the final manuscript.

\section{Ethics approval and consent to participate}

Appropriate permission was obtained from the commune authorities and local households before the collection of parasite specimens from their stocks.

\section{Consent for publication}

Not applicable.

\section{Competing interests}

The authors declare that they have no competing interests.

\section{Publisher's Note}

Springer Nature remains neutral with regard to jurisdictional claims in published maps and institutional affiliations.

\section{Author details}

${ }^{1}$ National Institute of Veterinary Research, 86. Truong Chinh Street, Dong Da District, Hanoi, Vietnam. ${ }^{2}$ Department of Biomedical Sciences, Institute of Tropical Medicine, Nationalestraat 155, B2000 Antwerp, Belgium.

${ }^{3}$ Department of Virology, Parasitology and Immunology, Faculty of Veterinary Medicine, Ghent University, 133 Salisburylaan, B9820, Merelbeke, Belgium. ${ }^{4}$ Department of Veterinary Public Health and Food Safety, Faculty of Veterinary Medicine, Ghent University, 133 Salisburylaan, B-9820 Merelbeke, Belgium. ${ }^{5}$ Department of Parasitology, Faculty of Veterinary Medicine, Vietnam National University of Agriculture, Trau Quy, Gia Lam, Hanoi, Vietnam. ${ }^{6}$ Department of Immunology, Institute of Biotechnology and Graduate University of Science and Technology, Vietnam Academy of Science and Technology, 18. Hoang Quoc Viet Rd, Cau Giay, Hanoi, Vietnam.

Received: 16 June 2017 Accepted: 31 October 2017

Published online: 21 November 2017

References

1. Scholz T. Family Opisthorchiidae Looss, 1899. In: Bray RA, Gibson DI, Jones A, editors. Keys to the Trematoda, Vol. 3. Wallinford: CAB international and the Natural History Museum; 2008. p. 9-49.

2. Petney TN, Andrews RH, Saijuntha W, Wenz-Mücke A, Sithithaworn P. The zoonotic, fish-borne liver flukes Clonorchis sinensis, Opisthorchis felineus and Opisthorchis viverrini. Int J Parasitol. 2013:43(12-13):1031-46.

3. Doanh PN, Nawa Y. Clonorchis sinensis and Opisthorchis spp. in Vietnam: current status and prospects. Trans R Soc Trop Med Hyg. 2016;110(1):13-20.

4. Dao TT, Bui TV, Abatih EN, Gabriël S, Nguyen TT, Huynh QH, et al. Opisthorchis viverrini infections and associated risk factors in a lowland area of Binh Dinh Province, Central Vietnam. Acta Trop 2016;157:151-157.

5. Dao TH, Nguyen TG, Victor B, Gabriël S, Dorny P. Opisthorchis viverrini-like liver fluke in birds from Vietnam: morphological variability and rDNA/ mtDNA sequence confirmation. J Helminthol. 2014;88(4):441-6.

6. Dao HT, Abatih EN, Nguyen TT, Tran HT, Gabriël S, Smit S, et al. Prevalence of Opisthorchis viverrini-like fluke infection in ducks in Binh Dinh Province, Central Vietnam. Korean J Parasitol. 2016:54(3):357-61.

7. Nawa Y, Doanh PN, Thaenkham UI. Opisthorchis viverrini an avian liver fluke? J Helminthol. 2015:89(2):255-6.

8. Oshmarin PG. Helminths of animals of Southeast Asia. In: Oshmarin PG, Mamaev YL, Lebedev BI, editors. Trematodes of domesticated and wild birds in the Democratic Republic of Vietnam. Moscow: Nauka; 1970. p. 5126. (In Russian).

9. Le NT. [Fauna of Vietnam.] Hanoi: Science and Techniques Publishing House. (In Vietnamese); 2000. p. 236-46.

10. Tan ND, Thoai NV, Phuong TH. Development of egg and larvae of Opisthorchis parageminus in environment and in intermediate host. J Vet Sci Techn. 2017:3:58-63. (In Vietnamese with English abstract)

11. Dorny P, Dao T, Victor B, Nguyen T, Gabriël S. Response to manuscript 'Is Opisthorchis viverrini an avian liver fluke? J Helminthol. 2015;89(2):257-8.

12. Le TH, Nguyen KT, Nguyen NT, Doan HT, Dung DT, Blair D. The ribosomal transcription units of Haplorchis pumilio and $\mathrm{H}$. taichui and the use of $28 \mathrm{~S}$ rDNA sequences for phylogenetic identification of common heterophyids in Vietnam. Parasit Vectors. 2017:10(1):17.

13. Olson PD, Cribb TH, Tkach W, Bray RA, Littlewood DTJ. Phylogeny and classification of the Digenea (Platyhelminthes: Trematoda). Int J Parasitol. 2003;33:733-55

14. Kostadinova A, Pérez-del-Olmo A. The systematics of the Trematoda. In: Toledo F, Fried B, editors. Digenetic Trematodes. Advances in Experimental Medicine and Biology. New York: Springer Science + Business Media; 2014. p. 21-44.

15. Tkach W, Kudlai O, Kostadinova A. Molecular phylogeny and systematics of the Echinostomatoidea Looss, 1899 (Platyhelminthes: Digenea). Int J Parasitol. 2016;46:171-85. 
16. Shekhovtsov SV, Katokhin AV, Kolchanov NA, Mordvinov VA. The complete mitochondrial genomes of the liver flukes Opisthorchis felineus and Clonorchis sinensis (Trematoda). Parasitol Int. 2010;59(1):100-3.

17. Thaenkham U, Nawa Y, Blair D, Pakdee W. Confirmation of the paraphyletic relationship between families Opisthorchiidae and Heterophyidae using small and large subunit ribosomal DNA sequences. Parasitol Int. 2011;60(4):521-3.

18. Thaenkham U, Blair D, Nawa Y, Waikagul J. Families Opisthorchiidae and Heterophyidae: are they distinct? Parasitol Int. 2012;61(1):90-3.

19. Cai XQ, Liu GH, Song HQ, CY W, Zou FC, Yan HK, et al. Sequences and gene organization of the mitochondrial genomes of the liver flukes Opisthorchis viverrini and Clonorchis sinensis (Trematoda). Parasitol Res. 2012;110(1):235-43.

20. Dao HT, Dermauw V, Gabriël S, Suwannatrai A, Tesana S, Nguyen GT, Dorny P. Opisthorchis viverrini infection in the snail and fish intermediate hosts in Central Vietnam. Acta Trop. 2017;170:120-5.

21. Tamura K, Stecher G, Peterson D, Filipski A, Kumar S. MEGA6: Molecular Evolutionary Genetics Analysis version 6.0. Mol Biol Evol. 2013;30:2725-9.

22. Na L, Gao JF, Liu GH, Fu X, Su X, Yue DM, et al. The complete mitochondrial genome of Metorchis orientalis (Trematoda: Opisthorchiidae): comparison with other closely related species and phylogenetic implications. Infect Genet Evol. 2016;39:45-50.

23. Sripa B, Brindley PJ, Mulvenna J, Laha T, Smout MJ, Mairiang E, et al. The tumorigenic liver fluke Opisthorchis viverrini - multiple pathways to cancer Trends Parasitol. 2012;28:395-407.

24. Lee D, Choe S, Park H, Jeon HK, Chai JY, Sohn WM, et al. Complete mitochondrial genome of Haplorchis taichui and comparative analysis with other trematodes. Korean J Parasitol. 2013;51(6):719-26.

25. Le TH, Blair D, McManus DP, Complete DNA. Sequence and gene organization of the mitochondrial genome of the liver fluke, Fasciola hepatica L. (Platyhelminthes; Trematoda). Parasitology. 2001;123(6):609-21.

26. Liu GH, Gasser RB, Young ND, Song HQ, Ai L, Zhu XQ. Complete mitochondrial genomes of the 'intermediate form' of Fasciola and Fasciola gigantica, and their comparison with F. hepatica. Parasit Vectors. 2014;7:150.

27. Ma J, Sun MM, He JJ, Liu GH, Ai L, Chen MX, Zhu XQ. Fasciolopsis buski (Digenea: Fasciolidae) from China and India may represent distinct taxa based on mitochondrial and nuclear ribosomal DNA sequences. Parasit Vectors. 2017;10(1):101.

28. Ma J, He JJ, Liu GH, Leontovyč R, Kašný M, Zhu XQ. Complete mitochondrial genome of the giant liver fluke Fascioloides magna (Digenea: Fasciolidae) and its comparison with selected trematodes. Parasit Vectors. 2016;9(1):429.

29. Littlewood DT, Lockyer AE, Webster BL, Johnston DA, Le TH. The complete mitochondrial genomes of Schistosoma haematobium and Schistosoma spindale and the evolutionary history of mitochondrial genome changes among parasitic flatworms. Mol Phylogenet Evol. 2006;39(2):452-67.

30. Thaenkham U, Dekumyoy P, Komalamisra C, Sato M, Dung do T, Waikagul J. Systematics of the subfamily Haplorchiinae (Trematoda: Heterphyidae), based on nuclear ribosomal DNA genes and ITS2 region. Parasitol Int. 2010;59(3):460-5.

31. Pornruseetairatn S, Kino H, Shimazu T, Nawa Y, Scholz T, Ruangsittichai J, et al. A molecular phylogeny of Asian species of the genus Metagonimus (Digenea) - small intestinal flukes - based on representative Japanese populations. Parasitol Res. 2016;115(3):1123-30.

32. Le TH, Nguyen VD, Phan BU, Blair D, McManus DP. Case report: unusual presentation of Fasciolopsis buski in a Vietnamese child. Trans R Soc Trop Med Hyg. 2004;98(3):193-4

33. Lotfy WM, Brant SV, DeJong RJ, Le TH, Demiaszkiewicz A, Rajapakse RP, et al. Evolutionary origins, diversification, and biogeography of liver flukes (Digenea, Fasciolidae). Am J Trop Med Hyg. 2008;79(2):248-55.

34. Johnston DA, Kane RA, Rollinson D. Small subunit (18S) ribosomal RNA gene divergence in the genus Schistosoma. Parasitology. 1993;107(2):147-56.

35. Lockyer AE, Olson PD, Ostergaard P, Rollinson D, Johnston DA, Attwood SW, et al. The phylogeny of the Schistosomatidae based on three genes with emphasis on the interrelationships of Schistosoma Weinland, 1858 Parasitology. 2003;126(3):203-24.

\section{Submit your next manuscript to BioMed Central and we will help you at every step:}

- We accept pre-submission inquiries

- Our selector tool helps you to find the most relevant journal

- We provide round the clock customer support

- Convenient online submission

- Thorough peer review

- Inclusion in PubMed and all major indexing services

- Maximum visibility for your research

Submit your manuscript at www.biomedcentral.com/submit
Biomed Central 